\title{
Combined experimental and numerical approach for identification of dynamic material model parameters
}

\author{
J. Peirs ${ }^{\mathrm{a}}$, P. Verleysen, W. Van Paepegem and J. Degrieck \\ Department of Materials Science and Engineering, Ghent University, 9000 Gent, Belgium
}

\begin{abstract}
Extraction of the material stress-strain curve from a dynamic tensile or shear experiment is not straightforward. Indeed, stress and strain are not homogeneously distributed in the specimen, and consequently no one-one relation exists between the measured elongation and strain on one hand, and the measured force and stress on the other hand. This work aims at improving the accuracy of the stress-strain curves calculated from high strain rate experiments and the modelling of the material behaviour. Therefore numerical simulations are used to determine the relationship between the average stress-strain and local effective stress-strain. The material model parameters used in these simulations are improved during an iterative procedure which combines the experimental results and the simulated stress and strain distribution. Stress triaxiality, local temperature and strain rate are taken into account. The method is applied to dynamic tensile and shear experiments on a Ti6Al4V alloy carried out on a split Hopkinson bar set up. The Johnson-Cook model is used to describe the strain rate and temperature dependent material behaviour. The two types of tests are used separately or simultaneously to extract and model the material behaviour. It is found that using tensile and shear experiments simultaneously has clear advantages. The same approach is used to identify parameters for the Johnson-Cook damage initiation criteria.
\end{abstract}

\section{Introduction}

The split Hopkinson bar technique is well known for characterizing the high strain rate behaviour of materials. The specimen can be loaded in tension, compression, torsion, shear or bending. For tension and compression experiments, the possibility to determine the stress and strain history in the specimen without direct measurement on the specimen itself is generally acknowledged to be one of the main advantages of the Hopkinson technique. However, this is only possible if the condition of homogeneous stress and strain distribution in the specimen is fulfilled.

Recently, advanced full field strain measurements [1] have shown that the assumption of a homogeneous strain distribution is not obvious [2]. The average strain that is determined from the split Hopkinson bar records only corresponds with the local strain for specimens where the deforming section of the specimen is well defined. If significant deformation is taking place outside the gage section, and when necking develops, the strains calculated from the waves measured on the Hopkinson bars are not valid. In practice, for a number of materials, the measured average stress-

\footnotetext{
${ }^{\text {a } E-m a i l: ~ J a n . P e i r s @ U G e n t . b e ~}$
} 
strain curve is still a relatively good representation of the real material behaviour because the error made by not considering the strain outside the gage section is compensating for the error made by not considering the strain localization in the centre of the gage section. However, the accuracy of the obtained strain is material dependent; for materials with a low strain hardening such as Ti6Al4V the strain reached in the specimen is seriously underestimated. The strain distribution does not only depend on the specimen geometry, which varies during the experiment, but also on the materials constitutive behaviour. This should be kept in mind when comparing experimental stress-strain curves of different materials. In addition, full field strain measurements have revealed the existence of a more complex strain state than typically considered in the tests.

In order to identify the parameters of dynamic constitutive models such as the Zhao, Ludwig or Johnson-Cook model, the effective stress - effective strain behaviour as function of the strain rate and temperature should be known accurately. Unfortunately, the split Hopkinson bar tests provide no more than the average stress and strain in one direction. The obtained data contains both the average material behaviour and the structural specimen behaviour. In the last decades, several approaches for extracting the local material behaviour from the global specimen response have been developed.

Early methods make use of analytical expressions such as the method from Bridgman [3] does for the geometry of the diffuse neck. More recently developed techniques make use of finite element simulations. These methods [4,5] typically involve an iterative optimization of the material model by minimizing the difference between the simulated and experimental load-displacement curves. An alternative approach to obtain more accurately the local strain in the specimen uses optical (full field) strain measurements such as the moiré phase shifting [6] and digital image correlation technique (DIC). Other advanced techniques for effective stress-strain determination are based on a combination of FE simulations and DIC [7,8]. All previously listed methods have mainly been applied on quasi-static tensile tests. Literature on identification methods for dynamic material behaviour and other than tensile load configurations is rare.

In this work, an alternative iterative experimental-numerical method is presented to extract the local high strain rate material behaviour (effective stress and strain) of Ti6Al4V in the centre of the specimen. The method takes into account non-axial strain components and the distribution of the stress, strain and temperature in the specimen without the need of digital image correlation. Application is not restricted to only dynamic tensile tests but also shear tests can be used. The method is very suited to accurately fit material model parameters to the experimental behaviour. In this work the Johnson-Cook model is used but other models could be used either. The Johnson-Cook parameters that are obtained during this procedure are more realistic than with methods that only use the average stress-strain curves because local adiabatic temperature, strain rate and stress triaxiality are taken into account during the parameter fitting process. The information from the FE simulations is also applied to determine parameters from damage initiation criteria such as the Johnson-Cook failure criterion.

The use of both tensile and shear experiments for fitting of the constitutive model, leads to model parameters that are applicable for a wider range of loading paths. For damage initiation criteria that take into account the effect of stress triaxiality it is even inevitable to use different load configurations. Furthermore, comparison of the results from the two experimental techniques extends the insight in the effect of the specimen geometry on the results.

The first part of the paper describes the experimental techniques and obtained results. The second part of the paper focuses on the material behaviour extraction method and identification of the parameters from the constitutive and damage initiation model.

\section{Experimental observations}

High strain rate tensile as well as shear tests can be carried out on the split Hopkinson tensile bar set up. A dogbone shaped specimen (Figure 1) is used for the tensile experiments, while a purposedeveloped specimen geometry (Figure 2) is used for the shear experiments. The specimen geometry is optimized for low stress triaxiality and applicability in dynamic tests [9]. The specimens are cut by 
means of electrical discharge machining from the same $0.6 \mathrm{~mm}$ thin sheet of Ti6Al4V. Both specimen types have a similar size and are glued in the slits of the Hopkinson bars resulting in a fixture with low interference with the stress waves.

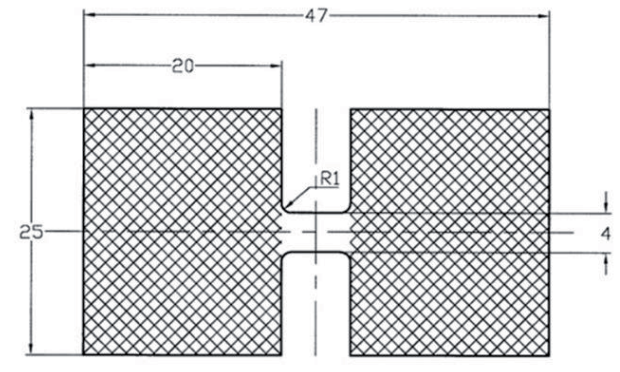

Fig. 1. Dogbone shaped tensile specimen

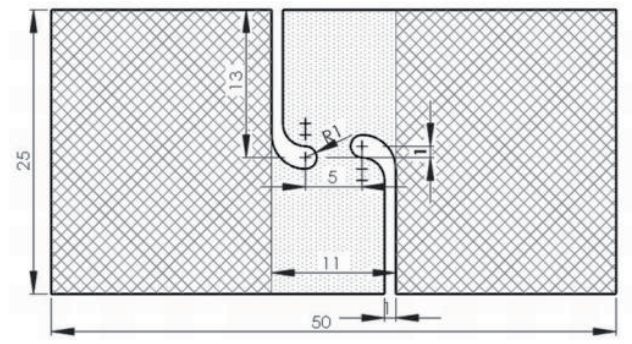

Fig. 2. Planar shear specimen

Dynamic tensile tests are carried out on specimens in the rolling (RD) and transverse (TD) direction. The average true stress-strain curves using the assumption of a homogeneous stress and strain distribution are shown in Figure 3 [10]. For one experiment the strain measured with DIC is used instead of the strain determined from the Hopkinson bar waves. This corrected stress-strain curve is also shown on Figure 3 for comparison. Two important aspects on the behaviour of Ti6Al4V can be seen. First, the Ti6Al6V shows low strain hardening and is therefore expected to be prone to strain localization. Second, the local strain in the centre of the specimen measured with DIC is much higher than the average strain derived from the Hopkinson wave records, supporting the first conclusion. The high strain has also been checked with post-mortem analysis of the specimen. Because the actual strain is higher than the average strain, also the actual true stress is higher than the average one. From this it can be concluded that the average stress-strain curves are not a good description of the real material behaviour.

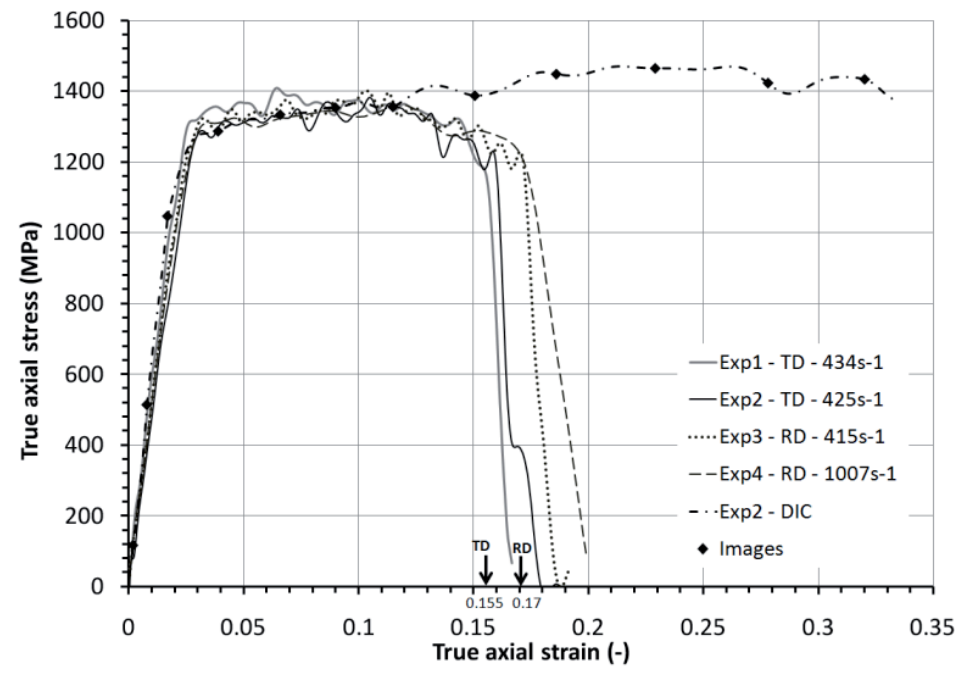

Fig. 3. True stress-strain curves obtained by Hopkinson bar records and DIC

The shear tests allow studying the material behaviour at low stress triaxiality. During shear deformation there is no cross section reduction which can lead to a more stable deformation. The relation between true and engineering values of the stress is much simpler. Furthermore, damage growth is retarded because of the lower stress triaxiality in shear. Dependent on the material, the shear test can thus be used to characterize the behaviour at larger strains than in a tensile test [11]. On the other hand, uniform deformation can be interrupted abruptly if the material is susceptible to 
shear localization, which is the case for Ti6Al4V. The effect of the structural specimen behaviour on the outcome of the experiment is different and more complicated than in the tensile test. Local strain measurements or calculations are necessary because the plastic region of the shear specimen is even less well defined than for a tensile specimen.

\section{Extraction of constitutive material behaviour}

The local stress, strain, strain rate and temperature in the centre of the specimen are calculated by combining the experimental average stress-strain curves with information obtained from finite element simulations. On the one hand, the experiments provide the global force-displacement behaviour of the specimen. On the other hand, the FE simulations give the relation between the global force and displacement and the local stress and strain. This relation contains the material and structural specimen behaviour, and is thus dependent on the material model implemented in the FEM. The commercial code ABAQUS/Explicit is used for the FE simulations. The material behaviour is described by the Johnson-Cook (eq. 1) model but other models could be used as well.

$$
\sigma=\left[A+B \varepsilon_{p}^{n}\right]\left[1+C \ln \frac{\dot{\varepsilon}}{\dot{\varepsilon}_{0}}\right]\left[1-\left(\frac{T-T_{\text {room }}}{T_{\text {melt }}-T_{\text {room }}}\right)^{m}\right]
$$

$\dot{\varepsilon}_{0}$ is a reference value of the strain rate, $T$ the temperature, $T_{m e l t}$ the melting temperature and $T_{\text {room }}$ is the room temperature. The parameters $\mathrm{m}$ and $\mathrm{C}$ are adapted during successive iterations while the parameters A, B and $\mathrm{n}$ are determined by a static tensile test. Figure 4 illustrates how the material behaviour is extracted from the tensile and/or shear tests, the procedure is described hereunder.

Step 1: A dynamic experiment is carried out. For a tensile test, a first estimation of the material behaviour can be obtained by the classic assumptions of a homogeneous and uniaxial stress and strain distribution. Extraction of the material behaviour from the shear test is less obvious because the relation between the measured elongation of the specimen and the strain is not well known due to the more complex specimen geometry.

Step 2: A FE simulation of the dynamic experiments is performed. The material model used in this simulation can be based on the results of "Step1" or can come from literature. The first option is the most convenient for tensile tests but difficult to apply for the shear test or other non-standard tests. The following important relationships are deduced from the simulations:

A. The ratio of the total force $\mathrm{F}$ and local effective stress $\sigma$ in the centre of the specimen as function of the total specimen elongation $\Delta \mathrm{u}(\mathbf{A 1})$. For the tensile tests and shear tests, this ratio is almost equal to respectively the cross section of the specimen and the cross section of the specimen multiplied by $\sqrt{3}$.

B. The ratio of the total elongation $\Delta \mathrm{u}$ and local effective strain $\varepsilon$ in the centre of the specimen as function of the total specimen elongation $\Delta \mathrm{u}($ B1).

C. The local temperature and strain rate as function of the total specimen elongation $\Delta u(\mathbf{C} 1)$. The local strain rate and temperature are typically higher than the strain rate calculated from the Hopkinson waves and temperature calculated from the average strain. Temperature is calculated in the FE simulations by assuming adiabatic heating due to plastic work.

Step 3: Relationship A from "Step 2" is used to estimate the local stress in the centre of the specimen, based on the experimentally measured force. Relationship $\mathbf{B}$ is used for estimating the local strain, based on the experimentally measured elongation.

Step 4: New material model parameters are derived from the obtained local effective stress-strain curves and local temperature and strain rate data from "Step3". Optimization can be done with only the tensile tests, only the shear tests or both types of tests as presented on Figure 4.

Step 5: New simulations are performed with the material model calculated in the previous step. The simulation results are then compared with the experimental results. A second iteration can be done if the results are not satisfactory. The relations A2, B2 and $\mathbf{C 2}$ provided from the second 
simulation are more realistic than $\mathbf{A 1}, \mathbf{B} 1$ and $\mathbf{C} 1$ because the model parameters used in this simulation are more realistic than the original parameters.

Steps 2, 3 and 4 are repeated to increase the accuracy of the model. It is not needed to do a lot of iterations; a couple of iterations are sufficient to achieve good agreement between experiments and simulations. The parameters of the Johnson-Cook model after three iterations are presented in the table below.

Table 1. Extracted parameters for Johnson-Cook constitutive material model

\begin{tabular}{|c|c|c|c|}
\hline $\mathrm{A}=990 \mathrm{MPa}$ & $\mathrm{B}=767 \mathrm{MPa}$ & $\mathrm{n}=0.67$ & $\dot{\varepsilon}_{0}=0.0005 \mathrm{~s}^{-1}$ \\
\hline & $\mathbf{C}$ & $\mathbf{m}$ & \\
\hline Set3T & 0.024 & 0.57 & Optimized for tensile test $-3^{\text {nd }}$ iter. \\
\hline Set3S & 0.017 & 0.86 & Optimized for shear test $-3^{\text {nd }}$ iter. \\
\hline Set3TS & 0.019 & 0.70 & Optimized for tensile \& shear test $-3^{\text {nd }}$ iter. \\
\hline
\end{tabular}

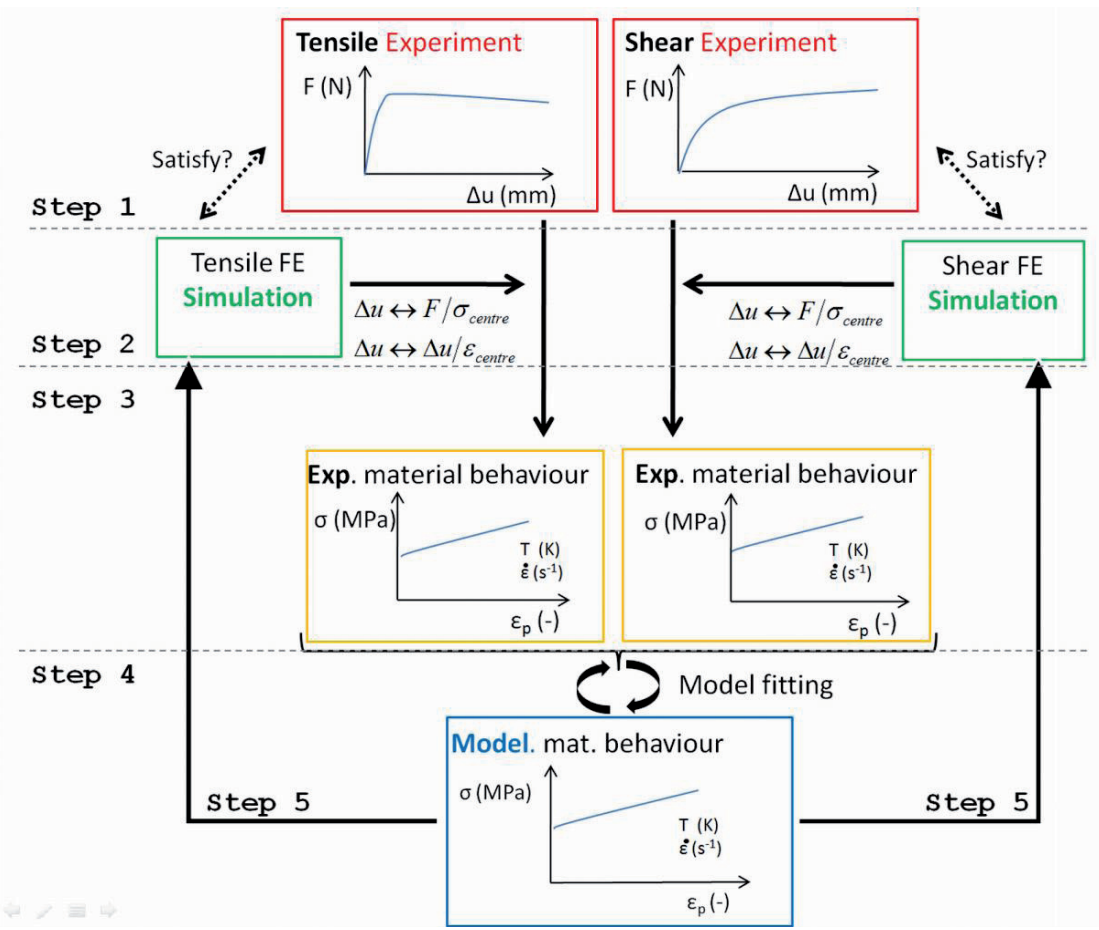

Fig. 4. Procedure to extract material behaviour and determine material model parameters from experiments by iterative extraction process

Figure 5 shows the local effective stress-effective strain curves extracted from the experimental results by use of numerical results after three iterations. The strain hardening and maximal strain presented on these corrected curves is clearly higher than presented on Figure 3 and corresponds much better with the experimental observations. In contrast with the force-displacement curves, the effective stress-effective strain curves from tensile tests are now comparable with those from shear tests. Thanks to this material behaviour extraction technique, the shear test can be used for characterizing the constitutive material behaviour.

The simulated and experimental engineering stress versus displacement curves for tensile and shear tests are compared in respectively Figures 6 and 7. There are three simulated curves for each experiment, dependent on the type of experiment(s) used to extract the material behaviour. It seems that the material model deduced from solely tensile tests (set $3 T$ ) or solely shear tests (set $3 S$ ) is 
appropriate for the simulation of respectively a tensile or shear experiment but not for both. Different material models are necessary to describe the material behaviour for different loading cases, which is a disadvantage. For a more complex loading case, a model that performs well for tensile and shear deformation is desired. Such model can be found by combining the shear and tensile experiments in the model fitting process (left and right side of Figure 4). Furthermore, it is easier to distinct the material behaviour from the global specimen response when the two test types are combined because the structural behaviour of the two specimen geometries is totally different but the material behaviour is almost the same. The JC model with parameter sets set3TS is a compromise between accuracy and general applicability of the model. Figures 6 and 7 demonstrate that these models give quite good results for both loading cases.

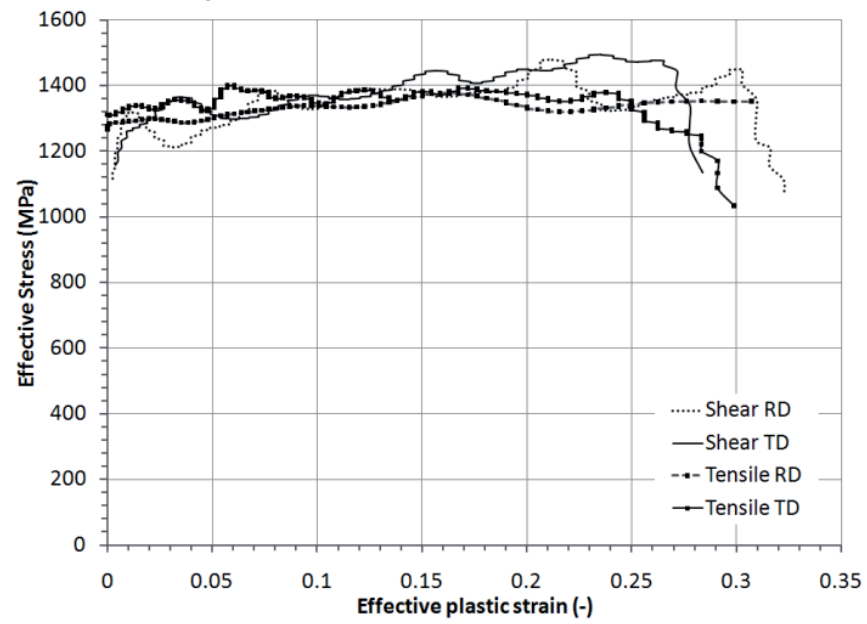

Fig. 5. Extracted experimental local effective stress-effective strain curves for two tensile and shear tests. Average local strain rate is approximately $1250 \mathrm{~s}^{-1}$.

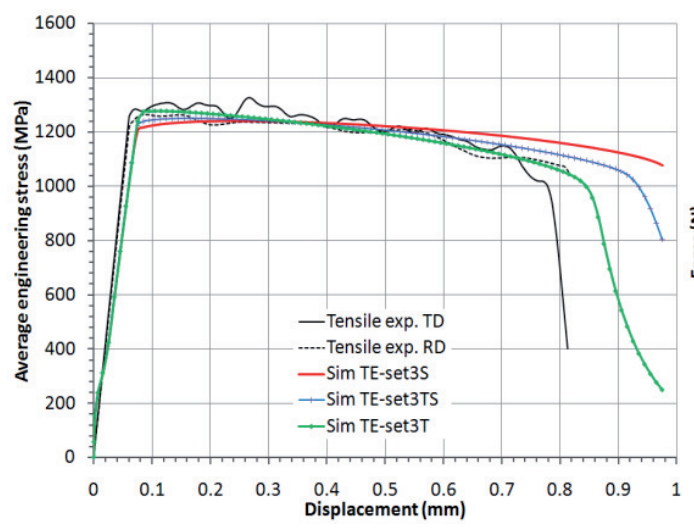

Fig. 6. Comparison of experimental and simulated eng. stress-displacement curves from tensile tests after $3^{\text {nd }}$ iteration

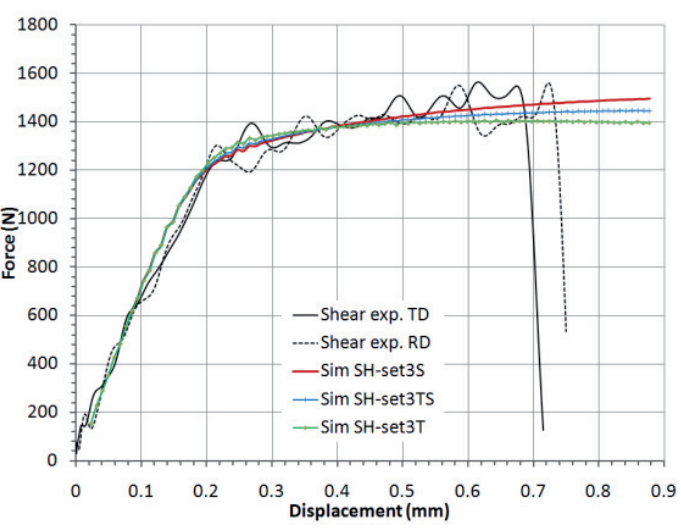

Fig. 7. Comparison of experimental and simulated force-displacement curves from shear tests after the $3^{\text {nd }}$ iteration

The material model and the FE simulation have been further evaluated by digital image correlation and measurement of the transverse contraction of the specimen during the test. It is found that the simulated strain distribution agrees well with the measured one, justifying the use of the FE model for extraction of the material behaviour. 


\section{Extraction of parameters for damage initiation criterion}

Next to the wider applicability, the use of multiple experiments with different stress triaxiality has the advantage of assessing the effect of triaxiality on the fracture strain. The the correct fracture strain in the tests can be determined with the material behaviour extraction method described earlier. Furthermore, the local temperature, strain-rate and stress triaxiality at the moment of fracture can also be obtained from the FE simulations. All this information is used to identify the parameters of a damage initiation criterion.

In this study, the Johnson-Cook ductile damage initiation criterion [12] is used. The phenomenological JC model does not take into account the physics of fracture and does not make a distinction between shear fracture and ductile fracture. Damage in the material is simply initiated when condition (2) is met. The equivalent plastic strain at onset of damage $\boldsymbol{\varepsilon}_{f 0}$ is calculated with expression (3).

$$
\begin{gathered}
\int \frac{d \varepsilon_{p}}{\varepsilon_{f 0}}=1 \\
\left.\boldsymbol{\varepsilon}_{f 0}=\left[d_{1}+\mathrm{d}_{2} \exp \left(-d_{3} n\right)\right]\left[1+d_{4} \ln \frac{\dot{\varepsilon}}{\dot{\varepsilon}_{0}}\right] 1+d_{5}\left(\frac{T-T_{\text {room }}}{T_{\text {melt }}-T_{\text {room }}}\right)\right]
\end{gathered}
$$

The parameters $d_{1}, d_{2}, d_{3}, d_{4}$ and $d_{5}$ are identified in a similar way as the parameters from the constitutive material model $(\S 3)$. A FE simulation is used to retrieve the stress triaxiality $n$, strain rate $\dot{\varepsilon}$ and temperature $T$ for each time increment at the location where fracture starts in the experiments. The damage initiation strain in each time step is then calculated with (3). The specimen elongation $\Delta \mathrm{u}_{\mathrm{f}}$, at which damage is initiated, is estimated from the experimental force-displacement curves. Next, the damage parameters are optimized to make the damage condition (1) true at that specific elongation $\Delta \mathrm{u}_{\mathrm{f}}$. This optimization is done simultaneously for a static tensile test, dynamic tensile test and dynamic shear test. The damage parameters that are obtained with the procedure are dependent on the constitutive material model used for the FE simulations of the tests. Therefore the damage initiation model will only work well when it is used together with the corresponding constitutive model.

Figure 3 shows the modelled effect of triaxiality on the damage initiation strain. High stress triaxiality leads to faster growth of voids and thus earlier ductile fracture.

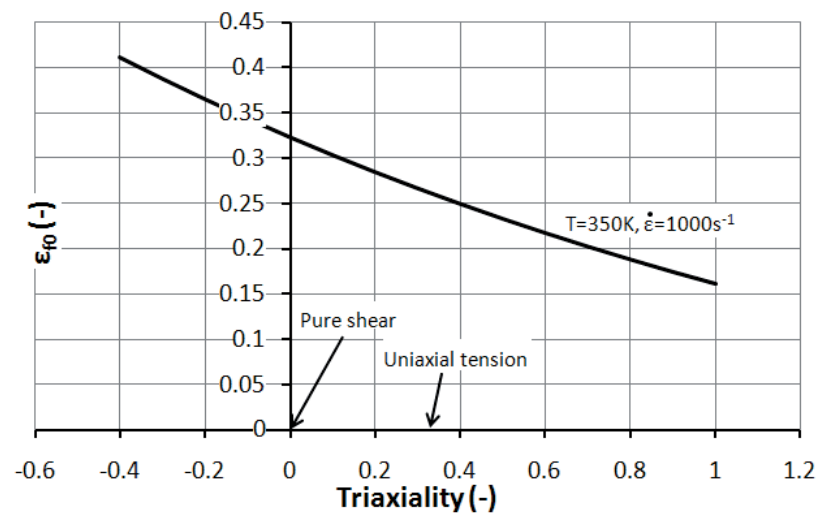

Fig. 3. Equivalent plastic strain at onset of damage as a function of stress triaxiality according to Johnson-Cook damage initiation criterion. Parameters for Ti6Al4V are: $\mathrm{d}_{1}=-0.05, \mathrm{~d}_{2}=0.183, \mathrm{~d}_{3}=0.452, \mathrm{~d}_{4}=0.078, \mathrm{~d}_{5}=3.87$. 


\section{Conclusions}

A combined experimental-numerical method to improve the extraction of the material behaviour from dynamic tensile and shear experiments was presented. One the one hand, the experiments provide the global force-displacement behaviour of the specimen. On the other hand, the simulations give the relation between the global force and displacement and the local stress and strain. The Johnson-Cook model is used in the FEM to describe the material behaviour. The parameters of that model are optimized for the experimental results by use of the simulated strain distribution itself. Therefore an iterative procedure is necessary to retrieve these parameters. The approach is applicable for different specimen geometries and makes the shear test appropriate for characterizing the constitutive material behaviour. It is shown that a similar approach can be used to determine parameters for the Johnson-Cook damage initiation criterion.

Using shear tests in addition to tensile tests yields clear advantages. It is found that the material model optimized for the tensile test results and shear test results simultaneously is more general applicable than a model that is derived from only tensile or only shear tests. In addition, the use of tests with different stress triaxiality is essential to identify the parameters for the Johnson-Cook damage initiation criterion.

\section{Acknowledgements}

The authors would like to acknowledge The Interuniversity Attraction Poles Program (IUAP) of the Federal Science Policy of Belgium and the partners of IUAP-VI (www.m3phys.be). The authors acknowledge the technical support of Dr. P. Lava and Dr. D. Debruyne from the Catholic University College Ghent for their in-house developed correlation software MatchID.

\section{References}

1. A. Gilat, T.E. Schmidt and A.L. Walker, Exp Mech, 49, 291 (2009)

2. P. Verleysen, J. Degrieck, T. Verstraete and J. Van Slycken, Exp Mech, 48, 587 (2008)

3. P.W. Bridgman, Studies in large plastic flow and fracture: with special emphasis on the effects of hydrostatic pressure (McGraw-Hill, N.Y., 1952)

4. K.S. Zhang and Z.H. Li, Eng Fract Mech, 49, 235 (1994)

5. M. Joun, J.G. Eorn and M.C. Lee, Mechanics of Materials, 40, 586 (2008)

6. P. Verleysen and J. Degrieck, Int J Impact Eng, 30, 239 (2004)

7. T. Hong, Z. Nian and T. Wei, Int J Mech Mater, 5, 13 (2009)

8. J. Kajberg and G. Lindkvist, Int J of Solids and Struct, 41, 3439 (2004)

9. J. Peirs, P. Verleysen, W. Van Paepegem and J. Degrieck, Proceedings of Dymat 2009, 1, 35 (2009)

10. H. Kolsky, Proceedings of the physical society, 62, 676 (1949)

11. V. Tarigopula, O.S. Hopperstad, M. Langseth, A.H. Clausen, F. Hild, O.G. Lademo and M. Eriksson, Exp Mech, 48, 181 (2008)

12. G.R. Johnson and W.H. Cook, Eng Fract Mech, 21, 31 (1985) 